\title{
Vinculándose al territorio: el ApS y la participación comunitaria. Estudio de caso en una escuela rural
}

Recibido: 17 de diciembre de 2018 / Revisado: 28 de enero de 2019

Aceptado: 26 de febrero de 2019 / Publicado: 22 de julio de 2019

CATERINA MONFORT MONFORT

Universitat Jaume I al376809@uji.es

AUXILIADORA SALES CIGES

Universitat Jaume I

asales@uji.es

http://dx.doi.org/10.24310/IJNE2.1.2019.6555

\section{RESUMEN}

La apertura de la escuela a la comunidad educativa se considera esencial para poder cubrir las necesidades sociales que esta tiene y para favorecer su democratización. En este estudio, realizado en el aula de sexto de uno de los dos aularios de un Colegio Rural Agrupado (CRA), se pretende conocer cuál es la percepción de la comunidad hacia un proyecto de Aprendizaje Servicio (ApS) de mejora de la escuela que se lleva a cabo en la clase y respecto su participación dentro del mismo. Se trata de un estudio de caso cualitativo que recoge la visión de dos madres, la tutora, la concejala de educación, el alumnado del aula de sexto, una vecina y dos alumnos de la UJI, a través de la técnica de la entrevista, la observación y el análisis documental. Los resultados muestran discrepancias entre la metodología del ApS y una más tradicional y una participación secundaria de la comunidad educativa. Como conclusión, se confirma la capacidad del ApS para vincular la escuela con agentes organizados del territorio, siempre y cuando la escuela tenga una cultura escolar de apertura a la comunidad.

Palabras Clave: Aprendizaje Servicio, relación escuela-comunidad, participación comunitaria.

\section{ABSTRACT}

Binding the school to the territory: the ApS and community participation. Case study in a rural school

The school opening to the community is considered essential in order to cover its social needs and to favour its democratization. This study, carried out in an Associated Rural School (CRA), is intended to find out what the perception of the community about a Service Learning (ApS) project is to improve the school and also to know about their participation in it. It is a qualitative case study that collects the vision of two mothers, the tutor, the councilor of education, the students of the sixth form class, a neighbor and two students of the Universitat Jaume I (UJI), through the technique of the interview, the observation and documentary analysis. The results show discrepancies between the ApS methodology and a more traditional one and a minor participation of the educational community. In conclusion, the ability of the ApS to link the school with organized agents of the territory is confirmed, as long as the school has a school culture of openness to the community.

Keywords: Service Learning, school-community relationship, community participation. 


\section{Introducción}

La apertura de la escuela a la comunidad educativa se considera una práctica fundamental para convertir el centro escolar en un espacio donde poder crecer y transformar el propio entorno, más allá del aprendizaje académico del alumnado. En las próximas líneas se hace un acercamiento a la cuestión de la participación de la comunidad a la escuela, se presenta la metodología de Aprendizaje Servicio (ApS) como una posible herramienta para favorecerlo y se plantean las dificultades que pueden darse.

Un estudio realizado por el Ministerio de Educación, Cultura y Deporte (MECD) destaca la importancia de que la escuela no esté de espaldas a la sociedad y a sus necesidades y requerimientos, principalmente porque una forma el futuro de la otra mediante un servicio que le realiza (MECD, 2014).

En esta línea, Zafra (2007, pp. 108-109) añade que “los sistemas escolares actuales [...], de la forma como están concebidos, no pueden hacer frente a todos los problemas que día a día entran por sus puertas", de forma que se necesita a la comunidad educativa para formar "a los ciudadanos del futuro", coincidiendo con aquello que plantea el estudio del MECD.

Parcerisa y Úcar (2007, p. 7) defienden la cuestión de la participación de la comunidad advirtiendo que "la escuela de nuestro tiempo tiene la necesidad de abrirse a la comunidad y romper con su tradicional aislamiento. Sin esta apertura difícilmente podrá cumplir la función social que tiene asignada”; haciendo referencia a la tarea que el centro escolar tiene de trabajar a través de redes con el territorio para que aquello que suceda en el aula adquiera sentido al relacionarse con la vida cotidiana.

Por su parte, aseguran que la comunidad tiene que contar con la escuela para hacer un proyecto comunitario, "como agente educador y, al mismo tiempo, como sujeto que se educa" (Parcerisa y Úcar, 2007, p. 8), remarcando el papel del agente externo al aula que también es responsable de la educación pero que tiene derecho a crecer dentro de este espacio, y sobre todo poniendo de manifiesto la necesidad de trabajar conjuntamente para introducir mejoras en un territorio que es compartido.

Traver, Sales y Moliner (2010) añaden que ante la sociedad del siglo XXI, y por lo tanto de la realidad de la globalización, la escuela tiene que ser un espacio en el cual formar a la ciudadanía desde una visión de comunidad intercultural y democrática para garantizar la reconstrucción social. Incluso se considera que este modelo organizativo podría servir de ejemplo para el funcionamiento de otras instituciones sociales.

De forma resumida, en realidad se está hablando de aquello que denominan escuela incluida (Traver, Sales, Moliner, Sanahuja y Benet, 2018), es decir, de un espacio que, asentado en los principios de la inclusión, la interculturalidad y la democracia, está dispuesto a trabajar conjuntamente con su comunidad para transformar las dinámicas del territorio en el cual se encuentra. 
Pero, cuando se habla de apertura a la comunidad educativa, ¿a qué grupo concreto se hace referencia? Para Moliner, Traver, Ruiz y Segarra (2016, p. 122), este grupo son “los agentes educativos que se encuentran en el contexto de la escuela". Y el territorio sería el espacio - el barrio, la localidad-, a pesar de que ahora desde una visión más dinámica puesto que no sólo es "el espacio por el cual se transita y se desarrollan nuestras acciones cotidianas, es también un entorno educativo” (Jiménez y Pozuelos, 2001, p. 13).

Por un lado, si se centra la atención en las familias de la escuela, un estudio realizado por la Universidad de Salamanca, considera que la participación de las familias a la escuela mejora las notas, supone actitudes más buenas hacia las tareas escolares, favorece una autoestima más elevada del alumnado e implica la realización de los deberes de una manera más perseverante (Calvo, Vergudo y Amor, 2016).

Además, tiene efectos positivos de cara a la docencia puesto que favorece su satisfacción con la propia profesión y supone mayor compromiso con la instrucción, ahora más centrada con el alumnado. En el caso de las familias, favorece la comprensión de los programas escolares, mejora la comunicación con los/las hijas y supone una mayor valoración de su papel en la educación (Calvo, et. al, 2016).

Hay que destacar que la presencia de estas dentro del contexto escolar "consolida la democratización de la escuela como comunidad donde es posible el intercambio de opiniones que contribuyen a promover buenas relaciones" (Payà y Tormo, 2016, p. 233). Por lo tanto, se trata de una participación que involucra a todas las personas que forman parte de la comunidad educativa generando una ciudadanía activa y comprometida con la mejora.

No obstante, a pesar de que la participación de las familias supone un derecho fundamental, habitualmente no existe una participación igualitaria entre los diferentes grupos integrantes de la comunidad escolar, reduciéndose la presencia de estas a los Consejos Escolares, y siendo como máximo informadas y consultadas (Silveira, 2016).

Por otro lado, si se centra la atención con otros agentes de la comunidad educativa, como pueden ser el Ayuntamiento o el vecindario, su participación, igualmente importante y beneficiosa, todavía suele ser menor. Además, "cuando esto tiene lugar muchas cosas cambian en la dinámica y el funcionamiento de la escuela. La regulación del centro tiene que variar, tiene que flexibilizarse”, a diferencia de cuando lo hacen las familias puesto que habitualmente se suelen instalar en las dinámicas ya existentes en el centro (Traver et. al., 2010, p. 109).

Por suerte, existen metodologías que favorecen esa participación comunitaria tan costosa dentro de espacios de educación reglada. Un ejemplo puede ser el Aprendizaje Servicio que consiste en la combinación de un servicio a la comunidad con el aprendizaje a través de la instrucción académica (Puig, Gijón, Martín y Rubio, 2011). 
Y es que, uno de los objetivos de esta metodología es favorecer la adquisición de contenidos curriculares de una forma más significativa: "los contenidos no aparecen de manera fragmentada y descontextualizada, sino que se vinculan con las necesidades de la realidad y se orientan a la acción" (Puig et. al., 2011, pp. 55-56), fomentando así la adquisición por parte del alumnado de un compromiso de mejora del propio entorno.

Pero, ¿se puede asegurar que el ApS favorece esa vinculación con el territorio sólo con la realización de un servicio? La realidad es que una de las características de la metodología descrita es que no puede llevarse a cabo de forma aislada ya que se necesita de todo un trabajo coordinado con otros agentes, es decir, necesita de redes locales para su buen funcionamiento.

Aún así, los resultados no son inmediatos. El hecho de aplicar una metodología como la mencionada no implica que la participación comunitaria esté asegurada puesto que el alumnado siempre suele estar en el centro y el hecho de abrir la escuela a otros agentes es visto como un peligro ya que podría dificultar el funcionamiento de las estructuras del centro (Traver et. al., 2010).

Además, aunque la escuela esté abierta a esa participación depende de muchos factores. De este modo, autores como Garreta (2008) apuntan a la falta de tiempo por parte de la comunidad para conciliar su vida laboral con la participación, aunque esto está determinado por el compromiso social con aquello que pasa dentro del centro escolar.

También tiene que ver qué visión se tiene de la cultura escolar, es decir, si se trata de un lugar exclusivo para el alumnado o de un espacio más amplio con el cual poder crecer y transformar el propio entorno. Respecto este hecho autores como Mérida (2002) apuntan a una posible sensación de inferioridad por parte de agentes educativos externos al aula que dificulta esa participación.

El presente estudio indaga sobre la percepción de la comunidad educativa ante un proyecto ApS de mejora de la escuela llevado a cabo en la clase de sexto de uno de los dos aularios de un Centro Rural Agrupado (CRA).

\section{Metodología: estudio de caso}

Siguiendo a Stake (1999), cuando se hace un estudio de caso se centra la atención en un caso singular que por algún motivo u otro es interesante, y se estudia la particularidad y complejidad del mismo, con la intención de comprenderlo. En el presente estudio, la elección de la metodología se debe de a la posibilidad de, como psicopedagoga, poder profundizar la realidad a partir de un contacto directo con la misma (Angulo y Noriega, 2014). 


\subsection{Contexto}

El contexto donde se ha centrado el estudio de caso es un CRA. Esta escuela rural se caracteriza por tener dos aularios, situados en dos pueblos diferentes. Hay que destacar que desde hace unos años este centro está implementando, junto al grupo de investigación MEICRI (Mejora Educativa y Ciudadanía Crítica) (UJI), un proyecto de transformación hacia una escuela más inclusiva e intercultural.

En este caso se pone el foco sólo en el aula de sexto de uno de los dos aularios, ya que el proyecto del otro aulario no se acaba de completar. En todo caso, en ambas clases durante el curso 2017/2018, se implementa un proyecto de ApS de mejora de la escuela, a partir del cual se pretenden abordar los contenidos curriculares establecidos, y vincular esto con un servicio a la comunidad puesto que es el propio alumnado quien diseña la escuela del futuro y busca los medios para hacer el proyecto realidad, a pesar de que abierto a la comunidad para que esta también pueda aportar y participar.

Es importante citar que el hecho de haber elegido la metodología ApS no es un hecho aislado sino que toma sentido a partir de una dinámica participativa realizada a las Jornadas de Puertas Abiertas de la escuela, o dicho de otra manera a la reunión que se realiza al inicio de curso, en la cual participa toda la comunidad educativa y se toma la decisión de trabajar en esa línea.

\subsection{Problema y preguntas de investigación}

Como ya se ha introducido, la metodología ApS ayuda a contextualizar los aprendizajes curriculares del aula con un servicio al entorno, que a la vez necesita de redes, en las cuales hay otros agentes de la comunidad. No obstante, centrando el foco en el aula de sexto del aulario objeto de estudio, no todas las personas de la comunidad creen que esta metodología alcanza los objetivos curriculares, tienen compromiso con aquello que pasa dentro de la escuela o han sido conocedoras del proyecto realizado.

Para comprender la complejidad de este caso se plantean dos preguntas. En primer lugar, Qué valor le da la comunidad educativa a la práctica de ApS frente a otras metodologías? En segundo lugar, Cómo ha visto la comunidad educativa su participación dentro de la ApS?

Por lo tanto, este estudio tiene dos objetivos a alcanzar:

1. Conocer la percepción de la comunidad educativa del aula de sexto sobre el valor del ApS para la educación de este alumnado.

2. Valorar la participación de la comunidad educativa en el proyecto ApS. 


\subsection{Participantes e instrumentos de recogida de información}

Las personas que participan de forma directa en el estudio son las familias de la clase (dos madres del aula), la tutora, el alumnado de sexto, una vecina y la concejala de educación del municipio. También se recoge información de dos alumnos en prácticas del Máster de Psicopedagogía (UJI), que recogen la información del día a día en un diario, y de las propias notas de campo de la investigadora.

La recogida de información ha supuesto la elaboración y uso de los instrumentos que se nombran y se explican a continuación:

- Técnica de la entrevista en forma de guiones de entrevista individuales semiestructurados, puesto que el guión es flexible. Se trata de dos bloques de cinco y seis preguntas respectivamente, titulados Visión respecto el ApS y Percepción respecto la participación de la comunidad educativa. Ésta información es grabada y posteriormente transcrita para su análisis. Concretamente, se entrevista a las dos madres del aula de sexto, a la concejala de educación y a la tutora. En el caso de las tres primeras, se les pregunta por la valoración que hacen del ApS llevado a cabo frente a una metodología más tradicional y por cómo han visto su participación dentro del proyecto y la del resto de personas de la comunidad. Para la tutora se mantiene el mismo esquema pero se le pregunta por su visión respecto la percepción de las personas externas al aula.

- Técnica de la observación en forma de registro anecdótico. Hay que destacar que la observación es sistemática ya que se observa atendiendo aquellos aspectos que previamente se planifica observar. Así, se toman como referencia los bloques citados en el apartado anterior y se formulan preguntas, dos en el primero y cinco en el segundo, a responder en una tabla en la que las columnas son los días y las filas las preguntas. Concretamente, se observa en cuatro días diferentes: el día que se entrevista a las dos madres, el día que se entrevista a la tutora, el día que se hace la reunión de planificación de la Jornada de Puertas Abiertas y el día de la Jornada de Puertas Abiertas. En cualquier caso, el registro se utiliza para observar a las familias, a personal del Ayuntamiento y a vecindario en relación a su percepción respecto el ApS y también para grabar su participación dentro de la escuela. Hay que remarcar que estas estancias en el centro se acompañan de notas de campo, a partir de las cuales se recoge la opinión de una vecina, y de fotografías y vídeos. Además, estos últimos son los que permiten grabar la visión del alumnado de sexto respecto la necesidad de hacer la ApS para mejorar la escuela.

- Técnica de análisis documental con diarios. Aquí también se toman como referencia los mismos bloques, esta vez de cuatro preguntas cada uno, y también se recoge la información en una tabla en la que cada columna corresponde a un diario y cada fila a una pregunta. Concretamen- 
te, se utilizan el diario de la tutora y el de los dos alumnos del Máster de Psicopedagogía de la UJI para conocer cómo viven el procedimiento del ApS las personas más cercanas al mismo. Con esta información también se quiere ver cuál es la importancia que estos agentes le dan a la comunidad educativa (sobre todo a las familias, el Ayuntamiento y el vecindario).

Finalmente, el hecho de haber elegido diferentes agentes de la comunidad educativa y haber hecho uso de diferentes instrumentos se debe de a la voluntad de construir una imagen más acercada a la realidad. De este modo, la intención al elegir estas fuentes e instrumentos es la de recoger diferentes perspectivas y por lo tanto la de triangular las fuentes de información (Jornet, Suárez y Perales, 2002).

Los instrumentos utilizados y las persones participantes presentan la siguiente codificación:

Tabla 1. Codificación de las personas participantes y de los instrumentos de recogida de información

\begin{tabular}{|c|c|}
\hline INSTRUMENTO & CÓDIGO \\
\hline Guión de entrevista a madre 1 & Efam1 \\
\hline Guión de entrevista a madre 2 & Efam2 Etut \\
\hline Guión de entrevista a la tutora & Ereg \\
\hline Guión de entrevista a la concejala de educación & reganec \\
\hline Registro anecdótico de la observación a familias, personal del Ayuntamiento y vecindario & notcamp \\
\hline Notas de campo propias & fot \\
\hline Fotografías & vid \\
\hline Dídeos & diariprac \\
\hline Diario de la tutora & del alumnado de la UJI \\
\hline
\end{tabular}

\subsection{Análisis de los resultados}

El análisis de los datos cualitativos extraídos se ha hecho a través de la organización de la información en categorías (Rodríguez, Gil y García, 1996). Estas etiquetas han permitido clasificar los datos atendiendo los dos objetivos de la investigación. Concretamente, se ha hecho un análisis de contenido 
mixto: deductivo porque hay categorías previas (teóricas) e inductivo porque han emergido categorías nuevas de los datos (emergentes).

En primer lugar, se han generado dos categorías teóricas correspondientes a las dos preguntas del estudio y se les ha asignado un código, específicamente un número. En segundo lugar, atendiendo la información obtenida de las personas informantes y de los instrumentos utilizados, se han generado categorías emergentes dentro de las generales, a las cuales también se les ha otorgado un código, formado por un número seguido de otro número (González y Cano, 2010).

Como se ha podido ver a la tabla 1, también se han utilizado códigos para diferenciar la información aportada por cada fuente y para indicar el instrumento empleado en cada momento.

\subsection{Cuestiones éticas}

Antes de iniciar el estudio de caso se informa a las persones participantes de los objetivos de la presente investigación y se pide su consentimiento para formar parte de la misma. A lo largo de todo el procedimiento se guarda el anonimato de las personas que han participado y también la confidencialidad de la información extraída.

\section{Resultados}

Antes de empezar se presentan de forma numerada las principales categorías abordadas dentro de cada pregunta y las subcategorías en las cuales se han desglosado:

1. Visión respecto el ApS

1.1 Protagonismo del alumnado

1.2 Contenido curricular

1.3 Visión frente a la clase tradicional

1.4 ApS como herramienta para vincular la escuela con el territorio

2. Percepción sobre la participación de la comunidad educativa

2.1 Protagonismo de la comunidad educativa

2.2 Apertura de la escuela a la participación de la comunidad educativa

2.3 Implicación de la comunidad educativa

2.4 Difusión a la comunidad educativa del ApS

2.5 Relaciones entre diferentes agentes de la comunidad educativa 
A continuación, se presentan los principales resultados de este estudio, teniendo en cuenta los objetivos planteados al principio. Así, se describe el valor que se le dan a esas prácticas respecto otras metodologías y se detalla como se ha visto la participación de la comunidad educativa en el ApS. De forma resumida, los resultados muestran discrepancias entre la metodología del ApS y una más tradicional, y se concluye una participación secundaria de la comunidad.

\subsection{Qué valor le da la comunidad educativa a la práctica de ApS frente a otras metodologías}

El valor que las persones participantes le dan a la práctica del ApS frente a otras metodologías, generalmente, es muy positivo. En primer lugar, se considera que esta metodología pone en el centro al alumnado y le da un protagonismo (1.1.) que hace que este esté motivado y tenga ganas de aprender: "aprenden y no se dan cuenta de que están aprendiendo mucho" (Efam2). Un ejemplo de esto es cuando el alumnado canta una canción de forma divertida al patio ante toda la comunidad educativa justificando la importancia del ApS para poder cubrir las carencias que tiene el centro escolar (vid). No obstante, la tutora es consciente que esta forma de trabajar no se ajusta a los deseos de todas las familias puesto que se da por hecho que "algunas pensarán que no todo el alumnado puede participar igual" (Etut).

Aún así, una de las madres dice que "cuando tú tomas el rol de responsabilidad, de persona que investiga, el hecho de estar valorando esa información que tú has integrado y trabajando en esa información, ya te está haciendo que el aprendizaje sea muy significativo" (Efam1). Es decir, el hecho de trabajar con información que se puede aplicar a un caso real, favorece que se encuentre sentido a aquello que se hace en clase.

En segundo lugar, se asocia este protagonismo del alumnado con la manera con la que se trabaja el currículum (1.2.) a través del ApS al integrar las diferentes asignaturas al proyecto e incluso algunas habilidades sociales: “¡en qué nivel están trabajando! La parte de matemáticas, de geometría, etc. Y no sólo esto, aprender a escuchar, respetar, la generosidad, es un aprendizaje de vida” (Efam1).

No obstante, una vez más la tutora es consciente de que hay familias que no lo ven igual: "algunas familias pensarán que esto no vale para nada" (Etut). Hay que remarcar que una madre valora de forma positiva el hecho de haber obtenido información a principio de curso: "a principio de curso nos dan curricularmente qué tienen que hacer a lo largo del curso, los objetivos a alcanzar. Sí nos lo han demostrado” (Efam2) - se refiere a la inclusión del currículum reglado dentro del ApS - .

Y es que, en tercer lugar, el hecho de cambiar los libros de texto por el aprendizaje a través de proyectos genera controversia y da pie a hablar del ApS frente a una visión más tradicional (1.3.): "algunas familias van más por una vía tradicional; pero si hablamos de porcentajes hay más que piensan que la metodología del cole es buena y curricular, han aprendido a ver la formación personal del alumnado" 
(Etut). Así, una de las madres participantes comenta que "vengo de otra escuela tradicional. Hacen lo mismo e incluso más" (Efam2). En cambio, esta también explica las dificultades que ha tenido: "para mí lo más negativo ha sido que yo he ido perdida, porque no tenía libro de referencia" (Efam2).

Finalmente, se acaba valorando el ApS para vincular la escuela con el territorio (1.4.) y una madre resume la metodología como "comunidad educativa unida" (Efam1) o se comenta que "sí que lo favorece" (Efam2). No obstante, esta última madre cuenta que "mi hermana es de aquí y yo creo que si no fuera por mí no lo hubiera sabido. Se ha hecho difusión pero la gente no hace demasiado caso: hay que abrir la mente y confiar" (Efam2). También la otra madre cree que "el resto de familias tendrían que ver que también es de ellas, que es un proyecto que se quedará aquí, que es el futuro del cole" (Efam1).

Hay que destacar que en el momento en el cual se decide trabajar a través de esta metodología, según cuenta la concejala de educación "el 90\% del pueblo estaba a favor" (Ereg). En cambio, "en realidad lo que no gusta es que viene gente de fuera por esa forma de trabajar" (Ereg). Según esta afirmación, la metodología no acaba de vincular la escuela con el territorio: "este año se ha dado la desvinculación del pueblo. Anteayer se hizo un Consejo Escolar y la única persona del pueblo era yo" (Ereg).

\subsection{Cómo ha visto la comunidad educativa su participación dentro del ApS}

En primer lugar, en cuanto a la percepción que la comunidad educativa tiene sobre su protagonismo (2.1.) dentro del ApS, la concejala y las dos madres consideran que su participación ha sido "secundaria" (Efam2). Así, a los diarios tanto de la tutora como del alumnado de la UJI se destaca la importancia de la participación de la comunidad educativa pero prácticamente no se centra la atención en este aspecto - prima el protagonismo del alumnado- (diariprac, diraritut).

En cualquier caso, y en segundo lugar, que la participación de la comunidad educativa haya sido secundaria no quiere decir que la escuela no se haya abierto (2.2.) a la misma: "nos han dicho que podíamos entrar cuando quisiéramos" (Efam2). En esta línea, hay que destacar que a final de curso se hacen unas Jornadas de Puertas Abiertas para mostrar el trabajo hecho a las familias - y a quienes quieran del pueblo - y para demostrar que se han cumplido los objetivos (fot).

Pero, y en tercer lugar, ¿cuál ha sido la implicación de la comunidad educativa (2.3.) a lo largo del curso? Una de las madres participantes comenta que "vengo todos los jueves, me gusta y el trabajo me lo permite" (Efam2) o un padre y una madre acuden a la reunión de preparación de la Jornada de Puertas Abiertas y se ofrecen para explicar a toda la comunidad como el ApS incluye los contenidos curriculares establecidos (reganec). También se explica que "hicimos una difusión en el Ayuntamiento y fueron las 
familias de los y las niñas" (Etut). A pesar de que la otra madre reconoce que "por tema laboral no es tan fácil esa parte de colaboración" (Efam1).

Para revertirlo, la tutora asegura que "la ayuda hoy en día no tiene que ser una visita en la clase, se pueden hacer muchas cosas fuera" (Etut). Un ejemplo de esto es que "algunas han colaborado desde casa, muchas veces se puede ayudar directamente dando propuestas”, hecho que permite la vinculación de las familias con el proyecto del aula sin tener que estar dentro.

No obstante, se nombra cierta desvinculación: “no veo mucha voluntad por parte de las familias, no porque no nos hayan dado la oportunidad" (Efam2) o falta de motivación por el proyecto: "en las sesiones de planificación sólo vinieron tres, cuatro familias de catorce” (Etut). Sobre este hecho, la concejala de educación cree que "tiene que ver con los partidos políticos cien por cien, derecha e izquierdas, estos últimos estamos a favor" (Ereg) y una madre explica que ve "mucha diferencia entre las familias del pueblo y las de fuera: la gente no está abierta a este tipo de dinámica” (Efam2).

A todo esto, y en cuarto lugar, la tutora del aula objeto de estudio reconoce que la difusión (2.4.) hubiera podido ser mejor: "la información de la difusión no ha llegado" (Etut), a pesar de que una familia explica que "nos lo enviaban a través de vídeos" (Efam1). Por otro lado, una madre cree que respecto al vecindario la información "la tienen que conocer porque los maestros han hecho difusión pero no sé si son conscientes ni del trabajo que han hecho, ni de la repercusión que puede tener en el futuro" (Efam1). En esta línea, “una mujer que trabaja de limpiadora en la escuela y está escuchando la entrevista a una de las madres, al terminar dice que ella es del pueblo pero que la gente del municipio es muy cerrada” (notcamp).

Finalmente, y a pesar de todo lo expuesto anteriormente, se nombran ciertas relaciones entre agentes de la comunidad (2.5.): "veo una naturalidad, un acercamiento, que hay una relación bastante abierta con el Ayuntamiento" (Efam1), también se cuenta que "estaba la concejala, estaba el arquitecto, y estaba la alcaldesa, la sensación fue muy buena porque estaban involucrados realmente” (Efam2) - a la difusión en el Ayuntamiento- o "algunas familias han favorecido la conexión con la comunidad proporcionando contactos" (Etut).

\section{Discusión}

En primer lugar, en cuanto al primer objetivo Conocer la percepción de la comunidad educativa del aula de sexto sobre el valor del ApS para la educación de este alumnado, los resultados han demostrado que la metodología favorece el protagonismo del alumnado al incluir todas las asignaturas del currículum en un solo proyecto, que a la vez permite el trabajo de las habilidades sociales, a pesar de que 
esto implica discrepancias entre quien cree en la metodología y quien apuesta por una más tradicional, aspecto que se ve reflejado en un conflicto intercultural al propio territorio.

En cuanto al protagonismo que tiene el alumnado dentro del ApS, autores como Puig et. al. (2011) hablan de cómo esta metodología fomenta aquello que Delors (1996) consideraba como uno de los pilares fundamentales de la educación del siglo XXI: aprender a ser. Y esto se debe de a como el ApS favorece que el alumnado se libere de las propias limitaciones y ponga en juego todas las capacidades en una acción conjunta, hecho que le da mayor autonomía y responsabilidad.

Pero, la organización de la vida académica teniendo en cuenta el pilar mencionado, implica un cambio en la manera de aplicar el currículum. Respecto este hecho, Feito (2009) se pregunta si la escuela tiene que servir únicamente para pasar cursos y hacer exámenes o para que el alumnado aprenda a pensar. Respecto a los centros que priorizan este último objetivo comenta que:

"en estos centros se aprende aquello fundamental para la vida, se aprende a relacionarse con el resto, a reconocer las diferencias entre los seres humanos, el valor de la amistad y de la solidaridad. En definitiva, se aprende a ser buena persona, buen ciudadano y buen intelectual” (Feito, 2009, pp. 32-33).

En la línea de este último posicionamiento, Aubert y Garcia (2009) citan los principales juicios hechos por parte de la pedagogía crítica a la ideología del neoliberalismo al considerar que los centros educativos se encuentran al servicio de los mercados puesto que se convierte el discurso sobre la calidad educativa en la evaluación de resultados individuales o en el acceso posterior a un puesto de trabajo, y no en base a la transformación social que puede alcanzar la escuela.

Respecto esta cuestión, Torres (2001) añade que el material curricular de las escuelas tiene que despertar el interés del alumnado y generarle motivación, cuestión que inevitablemente mejora el clima de la clase al no existir el aburrimiento y, de manera más amplia, ayuda a ver muchas más posibilidades de intervención y de mejora de la sociedad en la cual viven y vivirán.

En cambio, Aubert y Garcia (2009) también son conscientes de que cuando se pone como objetivo el respecto a la diversidad del alumnado y a sus necesidades, muchas veces esto es incoherente con los buenos resultados académicos, y sobre todo suele afectar a los colectivos más desfavorecidos. A esta cuestión le ponen el nombre de currículum de la felicidad versus currículum de la competencia.

En cualquier caso, estas autoras apuntan directamente a la necesidad de diálogo entre las dos posturas, y apuestan exclusivamente por las comunidades de aprendizaje u otras experiencias educativas que comparten principios similares, como es el caso de la experiencia de ApS, puesto que favorecen el rendimiento académico al trabajar las asignaturas como un todo integrado en un proyecto aplicado al 
contexto, fomentando una educación para la vida, en la cual se considera fundamental la participación de la comunidad educativa (Aubert y Garcia, 2009).

Por lo tanto, y volviendo al proyecto ApS del aulario objeto de estudio, la valoración de su capacidad para vincular la escuela con el territorio tiene que tener en cuenta las dos posturas desglosadas - aunque la realidad es que entre el negro y el blanco hay mucha variedad de grises, simplemente es para favorecer la comprensión - para entender el conflicto existente en el territorio de la escuela entre quien apuesta por una educación basada en proyectos y quien prefiere una educación más tradicional.

Pero, respecto este caso, hay que ir más allá puesto que no solamente se trata de posiciones opuestas respecto qué metodología es mejor para el alumnado sino que también existe un conflicto intercultural entre quien acude al aulario exclusivamente por el tipo de dinámica que se da al mismo centro aunque no sea del municipio y quien es de la localidad, que ve atacada su identidad como pueblo.

En relación al tema del conflicto intercultural, Torres (2011) considera importante recordar que las identidades son siempre dinámicas y están condicionadas a modificarse en la medida en la que las personas se relacionan, básicamente porque somos seres sociales abiertos a aprender del resto y a transmitir.

No obstante, hay que tener en cuenta esta situación en el contexto de globalización actual, donde la tendencia es la creación de identidades homogeneizadas. Así, en algunos casos, esta situación mundial, lejos de generar mayor trabajo comunitario hacia los ataques mencionados, da lugar a un alto grado de miedo, que se traduce en comportamientos individualistas y cerrados frente a la diversidad, y sobre todo porque los medios de masas no hacen más que informar sobre delitos (Torres, 2011).

A pesar de todo, es inevitable citar que el simple hecho de cambiar la manera de trabajar el currículum en la escuela, en este caso a través de un servicio al pueblo de mejora de la escuela, implica una movilización del conocimiento generado al centro hacia el exterior, directa o indirectamente, en una sociedad en la que cada vez más escasea la democracia (Feito, 2009). Y es que como dicen Puig et. al., (2011, p. 48): "nos hacemos ciudadanos de una democracia en buena parte gracias a la educación", y en este caso la escuela podría ser un referente en cuanto a la valoración positiva de la diversidad para progresar hacia una escuela y una sociedad más inclusiva e intercultural (Traver et. al., 2010).

En segundo lugar, en referencia al segundo objetivo Valorar la participación de la comunidad educativa en el proyecto ApS, después de analizar la información recogida se ha visto que el protagonismo de la comunidad educativa ha sido secundario, a pesar de que la escuela se ha abierto a la participación de la misma, pero ha faltado implicación por parte de la comunidad y la difusión realizada por parte de la escuela generalmente no ha llegado, aunque sí se destacan algunas relaciones entre diferentes agentes de la comunidad como la escuela con el Ayuntamiento o los puentes creados entre algunas familias y la escuela, proporcionando contactos de referentes locales. 
Respecto a la participación de la comunidad educativa, autores como Jiménez y Pozuelos (2001) consideran que si la democracia escolar sólo se reserva a los aspectos formales de la escuela se acaba provocando el alejamiento de los agentes educativos externos al aula. En cambio, si se apuesta por su participación, no de forma puntual sino continua, con una actitud de apertura al entorno, considerando el desarrollo del centro como una responsabilidad y una consecución de todas las personas de la comunidad, su implicación y compromiso se harán más evidentes, y la escuela se podrá enriquecer de esas nuevas aportaciones.

En cambio, a pesar de que la escuela tenga una actitud de apertura, hay que tener en cuenta también en qué situación se suele encontrar la comunidad para participar, puesto que muchas veces no se tienen en cuenta sus necesidades y sólo se consideran las de la escuela. Ante esta situación, se recomienda contar con profesionales más allá de la docencia (Torres, 2011), como podrían ser los/las profesionales de la psicopedagogía, para que hagan ese trabajo de aproximación a otros agentes educativos de fuera del aula.

Además, es fundamental prestar atención a la manera como se difunde ese proyecto a toda la comunidad educativa, es decir, como se moviliza el conocimiento. En este punto habrá que tener en cuenta si la intención de la escuela es simplemente informar a la comunidad sobre los hallazgos o involucrarla para que ese conocimiento sea enriquecido por aportaciones de esta, y por lo tanto sea una vía para trabajar conjuntamente y no una participación puntual en la cual se transfiere información que habitualmente sólo tiene sentido para quien la genera (Perines, 2016).

$\mathrm{Y}$ es que, la difusión es fundamental porque es precisamente ese mensaje que se trabaja conjuntamente con el territorio, en el caso del ApS del aulario en el cual se ha puesto el foco en forma de valores participativos y de convivencia, que como se ha dicho en repetidas ocasiones hace que la escuela sea un modelo organizativo a seguir (Traver et. al., 2011), que incluso puede llegar a generar nuevas relaciones entre diferentes agentes de la comunidad, que seguramente supondrán nuevas ideas para mejorar el territorio, en el cual también se encuentra la escuela.

Para terminar, la vinculación de la escuela con el territorio es interesante que se haga con entidades de personas que ya están organizadas, ya que parten de la experiencia de trabajar ofreciendo servicios y por lo tanto conocen cuáles son las necesidades del entorno en el cual desarrollan sus acciones (Puig et al, 2011), orientando así los proyectos ApS que pueden surgir de la escuela y garantizando su éxito.

\section{Conclusiones}

La apertura de la escuela a la comunidad educativa es un hecho evidente que mejora el mismo centro escolar y el propio territorio, al trabajar por un objetivo común que es la mejora del bienestar de las 
personas que conviven mediante acciones conjuntas. No obstante, el hecho de relacionar a la escuela con la comunidad necesita de una cultura escolar participativa, que es aquella que forma parte de la escuela en movimiento, generando un aprendizaje enriquecido, frente a las escuelas estancadas, que dan lugar a un aprendizaje empobrecido (Rosenholtz, 1989).

No obstante, la línea de investigación que tiene que seguir este estudio es analizar la capacidad de transformación que puede llegar a tener la metodología del ApS a largo plazo en cuanto a la vinculación de la escuela con el territorio. Al realizarse el estudio durante la realización del proyecto no se ha podido recoger información alrededor de su impacto a lo largo del tiempo.

Ya acabando, el hecho de trabajar conjuntamente con la comunidad educativa no es un procedimiento fácil puesto que esto implica trabajar con la diversidad, en la cual surgen conflictos por la existencia de varias maneras de pensar, por suerte. Y es que, es precisamente esa variedad de puntos de vista la que hace posible ese progreso hacia otras formas de ser, vivir, hacer y habitar el mundo, aunque es imprescindible buscar unos valores mínimos comunes (Díaz-Aguado, 2003).

Finalmente, la vinculación de la escuela con el territorio necesita mayor atención por parte del centro escolar al papel que se le da a la comunidad, normalizando su estancia en la escuela como agente educativo que aporta su experiencia, pero también como aprendiz de todos los hallazgos que nacen de la escuela, y todo a través de un currículum participativo, intercultural e inclusivo, siguiendo la idea de escuela incluida. La experiencia de Aprendizaje Servicio favorece ese aprendizaje y trabajo conjunto ya que pone en juego la parte académica con un servicio al entorno, que al fin y al cabo es el espacio de vida compartido, que ahora también es educativo.

\section{Referencias bibliográficas}

Angulo, B. y Noriega, G. (2014). El estudio de caso: Alternativa de investigación en las Ciencias Sociales y Humanidades. Perspectivas docentes, 51, 13-22. Recuperado el 18 de septiembre de 2018 de: http://revistas.ujat.mx/index.php/perspectivas/article/view/1261/1010

Aubert, A. y García, C. (2009). La pedagogía crítica y el éxito académico de todos y todas. Teoría de la Educación. Educación y Cultura en la Sociedad de la Información 3(10), 231-242. Recuperado el 25 de septiembre de 2018 de: http://www.redalyc.org/ pdf/2010/201014898014.pdf 
Calvo, Ma . I., Verdugo, M. A. y Amor, A. M. (2016). La participación familiar es un requisito imprescindible para una escuela inclusiva. Revista Latinoamericana de Educación Inclusiva, 10(1), 99-113. Recuperado el 12 de septiembre de 2018 de: https://dialnet.unirioja. $\underline{\text { es/servlet/articulo? codigo }=5505135}$

Delors (1996). La educación encierra un tesoro. Madrid: UNESCO

Diáz-Aguado, M. J. (2003). Educación intercultural y aprendizaje cooperativo. Madrid: Pirámide

Garreta, J. (2008). La participación de las familias en la escuela pública. Las asociaciones de madres y padres del mundo. Madrid: CEAPA. Recuperado el 12 de septiembre de 2018 de: https://www.researchgate.net/publication/265217518 Garreta J La participacion de_las_familias_en_la escuela_publica_Las_asociaciones_de madres_y_padres_del_ alumnado CIDE CEAPA Madrid 2008

González, T. y Cano, A. (2010). Introducción al análisis de datos de investigación cualitativa: Tipos de análisis y proceso de codificación (II). Nure investigación, 45, 1-10. Recuperado el 18 de septiembre de 2018 de: http://www.nureinvestigacion.es/OJS/index.php/nure/ $\underline{\operatorname{article} / \text { view/476 }}$

Jiménez, J. R. y Pozuelos, F. J. (2001). Una escuela pública abierta a la comunidad. Investigación en la escuela, 33, 5-17. Recuperado el 12 de septiembre de 2018 de: https://idus.us.es/ xmlui/handle/11441/60324

Jornet, J., Suárez, J. y Perales, M. J. (2002). Guía Práctica para la Evaluación de Programas de Formación Ocupacional y Continua. València: Adeit

Mérida, R. (2002). Un espacio de encuentro entre la escuela y las familias: la escuela de madres y padres. Revista de Ciencias de la Educación, 192, 441-468. Recuperado el 12 de septiembre de 2018 de: http://redined.mecd.gob.es/xmlui/handle/11162/32528

Ministerio de Educación, Cultura y Deporte (2014). La participación de las familias en la educación escolar. Madrid: MECD. Recuperado el 12 de septiembre de 2018 de: https://www.mecd. gob.es/dam/jcr:8c2e037a-8673-4911-8594-d7aa12214d87/estudioparticipacion-pdf.pdf

Moliner, O., Traver, J. A., Ruiz, M. A. y Segarra, T. (2016). Estrategias que inciden en los procesos de democratización de la escuela. Una aproximación teórica. Revista Electrónica de Investigación Educativa, 18(2), 116-129. Recuperado el 14 de septiembre de 2018 de: https://redie.uabc.mx/redie/article/view/1110/1433 
Parcerisa, A. y Úcar, X. (2007). Introducción. En Castro, Ma . M., Ferrer, G., Majado, Mª . F., Rodríguez, J., Vera, J., Zafra, M. i Zapico, $\mathrm{M}^{\mathrm{a}}$. H. (Coords.), La escuela en la comunidad, la comunidad en la escuela (pp. 7-9). Barcelona: Graó

Payà, A. y Tormo, M. (2016). La participación educativa de las familias en una escuela pública valenciana. Un estudio cualitativo. Foro de Educación, 14(21), 227-248. Recuperado el 12 de septiembre de 2018 de: http://www.redalyc.org/pdf/4475/447546543012.pdf

Perines, H. (2016). Movilización del conocimiento en educación. Conexión entre la investigación, la política y la práctica: Una aproximación teórica, 1(10), 137-150. Recuperado el 1 de octubre de 2018 de: http://www.scielo.edu.uy/pdf/pe/v10n1/1688-7468pe-10-01-00137.pdf

Puig, J. M., Gijón, M., Martín, X. y Rubio, L. (2011): Aprendizaje-servicio y Educación a la ciudadanía. Revista de Educación, número extraordinario, 45-67. Recuperado el 13 de septiembre de 2018: http://www.revistaeducacion.educacion.es/re2011/re2011 03.pdf

Rodríguez, G., Gil, J. y García, E. (1996). Metodología de la investigación cualitativa. Granada: Aljibe

Rosenholtz, S. J. (1989). Teachers' Workplace: The Social Organization of Schools. Nova York: Longman

Silveira, H. (2016). La participación de las familias en los centros educativos. Un derecho en construcción. Revista Electrónica Universitaria de Formación del Profesorado, 19(1), 1729. Recuperado el 13 de septiembre de 2018 de: https://dialnet.unirioja.es/descarga/ articulo/5315531.pdf

Stake, R. E. (1999). Investigación con estudio de casos. Madrid: Morata. Recuperado el 18 de septiembre de 2018 de: https://www.uv.mx/rmipe/files/2017/02/Investigacion-con-estudios-de-caso.pdf

Torres, J. (2001). Educación en tiempos de neoliberalismo. Madrid: Morata

Torres, J. (2011). La justicia curricular: El caballo de Troya de la cultura escolar. Madrid: Morata

Traver, J. A., Sales, A. y Moliner, O. (2010). Ampliando el territorio: algunas claves sobre la participación de la comunidad educativa. REICE, Revista Iberoamericana sobre Calidad, Eficacia y Cambio en Educación, 8(3), 96-119. Recuperado el 14 de septiembre de 2018 de: http://www.redalyc.org/pdf/551/55115052007.pdf 
Traver, J. A., Sales, M., Moliner, M., Sanahuja, A. y Benet, A. (2018). Hacia una escuela incluida en su territorio: análisis de una práctica comunitaria. Edetania. Estudios Y Propuestas Socioeducativas, (53), 99-116. Recuperado el 14 de septiembre de 2018 de: http://revistas.ucv.es/index.php/Edetania/article/view/339

Zafra, M. (2007). Intervención escolar comunitaria en contextos multiculturales. En Castro, Mª . M., Ferrer, G., Majado, Mª . F., Rodríguez, J., Vera, J., Zafra, M. y Zapico, Ma. H. (Coords.), La escuela en la comunidad, la comunidad en la escuela (pp. 81-110). Barcelona: Graó 